\title{
Large-Scale Bayesian Logistic Regression for Text Categorization
}

\author{
Alexander GENKIN \\ DIMACS \\ Rutgers University \\ Piscataway, NJ 08854 \\ (alexgenkin@iname.com)
}

\author{
David D. LEWIS \\ David D. Lewis Consulting \\ Chicago, IL 60614 \\ (tmpaper06@DavidDLewis.com)
}

\author{
David MADIGAN \\ Dept. of Statistics \\ Rutgers University \\ Piscataway, NJ 08854 \\ (dmadigan@rutgers.edu)
}

\begin{abstract}
Logistic regression analysis of high-dimensional data, such as natural language text, poses computational and statistical challenges. Maximum likelihood estimation often fails in these applications. We present a simple Bayesian logistic regression approach that uses a Laplace prior to avoid overfitting and produces sparse predictive models for text data. We apply this approach to a range of document classification problems and show that it produces compact predictive models at least as effective as those produced by support vector machine classifiers or ridge logistic regression combined with feature selection. We describe our model fitting algorithm, our open source implementations (BBR and BMR), and experimental results.
\end{abstract}

KEY WORDS: Information retrieval; Lasso; Penalization; Ridge regression; Support vector classifier; Variable selection.

\section{INTRODUCTION}

Maximum likelihood logistic regression is a mainstay for statisticians. This approach enjoys a body of supporting theory and algorithms, features prominently in commercial statistical software, and its predictive accuracy is often competitive with that of newer techniques.

However, new applications have emerged that pose computational and statistical challenges to maximum likelihood logistic regression. In these applications the number of predictor variables is large ( $10^{4}$ and up) and usually exceeds the number of observations. Examples of such "short, fat" data sets include text categorization (our focus in this article), gene expression analysis, adverse event monitoring, longitudinal clinical trials, and some business data mining tasks.

The logistic regression model is often capable of providing highly accurate predictions in these applications. Computing the maximum likelihood fit of a logistic regression model on these data sets is often impossible, since standard software relies on matrix inversion. Even when this barrier is overcome, numerical ill-conditioning can result in a lack of convergence, large estimated coefficient variances, poor predictive accuracy, and/or reduced power for testing hypotheses concerning model assessment (Pike, Hill, and Smith 1980). Exact logistic regression suffers from many of the same problems (Greenland, Schwartzbaum, and Finkle 2000). Furthermore, although maximum likelihood has desirable asymptotic properties (Hadjicostas 2003), it often overfits the data, even when numerical problems are avoided.

Computational efficiency, both during fitting and when the fitted model is used for prediction, is also a problem. Most logistic regression implementations make use of matrix inversion, which in practice limits the number of predictor variables. Although feature selection (Kittler 1986; Mitchell and
Beauchamp 1988) reduces memory and computational requirements, it introduces new problems. First, the statistical foundation of most feature selection methods is unclear. This makes it difficult to, for instance, choose the number of features for a given task in a principled way. Second, the most efficient feature selection methods consider each feature in isolation and may choose redundant or ineffective combinations of features. Finally, it is typically unclear how to combine heuristic feature selection methods with, for instance, domain knowledge.

In this article we describe a Bayesian approach to logistic regression that avoids overfitting, has classification effectiveness similar to that of the best published methods, and is efficient both during fitting and at prediction time. The key to the approach is the use of a prior probability distribution that favors sparseness in the fitted model, along with an optimization algorithm and implementation tailored to that prior. By "sparseness," we mean that the posterior point estimates for many of the model parameters are zero.

We begin in Section 2 by describing how supervised learning is used in language processing, the motivating area for our work. In Section 3 we present the basics of our Bayesian approach to logistic regression, and in Section 4 we present the fitting algorithm. We describe the data sets and methods that we use in our experiments in Section 5, and give our experimental results in Section 6. We find that the sparse classifiers that we describe are competitive with state-of-the-art text categorization algorithms, including widely used feature selection methods. Finally, in Section 7 we present directions for future work.

(C) 2007 American Statistical Association and the American Society for Quality TECHNOMETRICS, AUGUST 2007, VOL. 49, NO. 3 DOI 10.1198/004017007000000245 


\section{TEXT CATEGORIZATION AND STATISTICAL LANGUAGE PROCESSING}

Text classification algorithms choose which of a set of classes to assign to a text. When those classes are of interest to only one user, we often refer to text classification as filtering or routing. When the classes are of more general interest (e.g., Library of Congress headings), we instead refer to text categorization.

The study of automated text categorization dates back more than 40 years (Maron 1961). In the last decade statistical approaches have dominated the research literature and, increasingly, operational practice. Statistical supervised learning approaches to text categorization induce ("learn") a classifier (i.e., a rule that decides whether or not a document should be assigned to a category) from a set of labeled documents (i.e., documents with known category assignments). Depending on the category scheme, a categorization task may be framed as one or more binary and/or polychotomous classification problems. Sebastiani (2002) provided a broad overview of statistical approaches to text classification.

Documents to be classified are typically represented as vectors of numeric feature values derived from words, phrases, or other characteristics of documents. The dimensionality of these vectors ranges from $10^{3}$ to $10^{6}$ or more. Early text categorization researchers therefore focused on learning algorithms that were both computationally efficient (for speed) and restricted in the classifiers that they could produce (to avoid overfitting). Examples include naive Bayes (Maron 1961; Lewis 1998) and the Rocchio algorithm (Rocchio 1971). Feature selection was often used to discard most features from the document vectors.

Greater computing power and new regularization approaches now allow learning of less-restricted classifiers both efficiently and with little overfitting. Example approaches include support vector machines (Joachims 1998; Zhang and Oles 2001; Lewis, Yang, Rose, and Li 2004), boosting (Schapire and Singer 2000), and ridge logistic regression (Zhang and Oles 2001). But, these methods still require selecting features and/or stopping fitting short of convergence if they are to produce compact (and thus efficient) classifiers.

Many of the characteristics of text categorization (e.g., short, fat data sets with murky relationships between features and class labels) are shared by other language processing applications. Indeed, as in text categorization, statistical techniques have displaced, or at least supplemented, the once-dominant knowledge engineering approach across all of computational linguistics. We briefly discuss other language processing tasks in which the approach described in this article might prove applicable.

As mentioned earlier, text categorization is just one of a range of text classification tasks. Supervised learning approaches have been applied to more personalized text classification tasks, such as sorting user e-mail and alerting users to news stories of interest. Filtering of text streams (for junk email, pornography, or proprietary information) straddles topical and personalized classification and in some cases must deal with an adversary attempting to evade classification (Madigan 2005). Authorship attribution (Mosteller and Wallace 1964; Madigan et al. 2005a; Madigan, Genkin, Lewis, and Fradkin 2005b) is an unusual classification task in which choosing training data, defining features, and even specifying the number of classes are tricky issues.

The foregoing applications treat documents as atomic units converted to feature vectors. Other tasks process language directly as a sequence of linguistic units. The goal may be linguistic analysis, such as finding the syntactic structure of a sentence or the meaning of an ambiguous word, or may be applicationoriented, such as finding all the names of companies in a set of documents. Still other tasks involve transducing language from one form to another, as in optical character recognition, speech recognition, machine translation, and summarization. A range of statistical approaches have been applied to such tasks, some that are explicitly sequential (many flavors of Markov models) and others that convert sequential data into vectors. Good texts on statistical language processing include those of Manning and Schütze (1999) and Jurafsky and Martin (2000). The use of text in data mining has been discussed by Weiss, Indurkhya, Zhang, and Damerau (2005) and Berry (2004).

\section{THE MODEL}

We are interested in learning classifiers, $y=f(\mathbf{x})$, from a set of training examples $D=\left\{\left(\mathbf{x}_{1}, y_{1}\right), \ldots,\left(\mathbf{x}_{i}, y_{i}\right), \ldots,\left(\mathbf{x}_{n}, y_{n}\right)\right\}$. For text categorization, the vectors $\mathbf{x}_{i}=\left[x_{i, 1}, \ldots, x_{i, j}, \ldots, x_{i, d}\right]^{T}$ consist of transformed word frequencies from documents (Sec. 5.1). The values $y_{i} \in\{+1,-1\}$ are class labels encoding membership $(+1)$ or nonmembership $(-1)$ of the vector in the category.

Concretely, we are interested in conditional probability models of the form

$$
p\left(y=+1 \mid \boldsymbol{\beta}, \mathbf{x}_{i}\right)=\psi\left(\boldsymbol{\beta}^{T} \mathbf{x}_{i}\right)=\psi\left(\sum_{j} \beta_{j} x_{i, j}\right) .
$$

In what follows we use the logistic link function

$$
\psi(r)=\frac{\exp (r)}{1+\exp (r)},
$$

thereby producing a logistic regression model.

For a text categorization problem, $p\left(y=+1 \mid \mathbf{x}_{i}\right)$ will be an estimate of the probability that the $i$ th document belongs to the category. The decision of whether to assign the category can be based on comparing the probability estimate with a threshold or, more generally, by computing which decision gives optimal expected utility.

For a logistic regression model to make accurate predictions for future inputs, we must avoid overfitting the training data. One Bayesian approach to avoiding overfitting involves a prior distribution on $\boldsymbol{\beta}$ specifying that each $\beta_{j}$ is likely to be near 0 . Next we describe several such priors.

\subsection{Gaussian Priors and Ridge Logistic Regression}

Perhaps the simplest Bayesian approach to the logistic regression model is to impose a univariate Gaussian prior with mean 0 and variance $\tau_{j}>0$ on each parameter $\beta_{j}$,

$$
p\left(\beta_{j} \mid \tau_{j}\right)=\mathrm{N}\left(0, \tau_{j}\right)=\frac{1}{\sqrt{2 \pi \tau_{j}}} \exp \left(\frac{-\beta_{j}^{2}}{2 \tau_{j}}\right), \quad j=1, \ldots, d .
$$


The mean of 0 encodes our prior belief that $\beta_{j}$ will be near 0 . The variances $\tau_{j}$ are positive constants that we must specify. A small value of $\tau_{j}$ represents a prior belief that $\beta_{j}$ is close to 0 . A large value of $\tau_{j}$ represents a less-informative prior belief. In the simplest case we let $\tau_{j}$ equal $\tau$ for all $j$. We assume a priori that the components of $\boldsymbol{\beta}$ are independent, and hence the overall prior for $\boldsymbol{\beta}$ is the product of the priors for each of its component $\beta_{j}$ 's. Finding the maximum a posteriori (MAP) estimate of $\boldsymbol{\beta}$ with this prior is equivalent to ridge regression (Hoerl and Kennard 1970) for the logistic model (Santner and Duffy 1989, sec. 5.4; le Cessie and van Houwelingen 1997).

This Gaussian prior, although favoring values of $\beta_{j}$ near 0 , does not favor the $\beta_{j}$ 's being exactly equal to 0 . Absent unusual patterns in the data, the MAP estimates of all $\beta_{j}$ 's will be nonzero. Previous authors have used feature selection to obtain sparse text classifiers with a Gaussian prior (Zhang and Oles 2001; Zhang and Yang 2003).

\subsection{Laplace Priors and Lasso Logistic Regression}

To produce a prior favoring sparse solutions, we again assume that $\beta_{j}$ arises from a Gaussian distribution with mean 0 and variance $\tau_{j}$,

$$
p\left(\beta_{j} \mid \tau_{j}\right)=\mathrm{N}\left(0, \tau_{j}\right), \quad j=1, \ldots, d .
$$

Further assume, a priori, that the $\tau_{j}$ 's arise from an exponential distribution with density

$$
p\left(\tau_{j} \mid \gamma\right)=\frac{\gamma_{j}}{2} \exp \left(-\frac{\gamma_{j}}{2} \tau_{j}\right), \quad \gamma>0 .
$$

Integrating out $\tau_{j}$ then gives an equivalent nonhierarchical double-exponential (Laplace) distribution with density

$$
p\left(\beta_{j} \mid \lambda_{j}\right)=\frac{\lambda_{j}}{2} \exp \left(-\lambda_{j}\left|\beta_{j}\right|\right)
$$

where $\lambda_{j}=\sqrt{\gamma}_{j}>0$. In what follows, we assume that $\lambda_{j}$ equals $\lambda$ for all $j$. As before, the prior for $\boldsymbol{\beta}$ is the product of the priors for its components. At 0 , the first derivative of this density is discontinuous. The distribution has mean 0 , mode 0 , and variance $2 / \lambda^{2}$.

Figures 1 and 2 show the effect of hyperparameter settings on the MAP logistic regression parameters on a particular data set with eight predictor variables. Figure 1 shows the effect of a Gaussian prior distribution on each parameter, with all Gaussians having the same variance. When that variance is small, the resulting MAP estimates are small, approaching 0 as the variance approaches 0 . When that variance is large, the MAP estimates are similar to the maximum likelihood estimates. The vertical dashed line corresponds to a variance of .01. Figure 2 shows the equivalent picture for the Laplace prior. As with the Gaussian prior, the MAP estimates range from all zeroes to the maximum likelihood estimates. However, unlike the Gaussian case, intermediate choices for the prior variance lead to MAP estimates where some components of $\boldsymbol{\beta}$ are 0 whereas others are not. The vertical dashed line corresponds to a variance of .27 , giving a posterior mode where two of the parameters are 0 . Hastie, Tibshirani, and Friedman (2001) showed similar plots for linear regression.

Tibshirani (1996) introduced the LASSO for linear regression as an alternative to feature selection for producing sparse

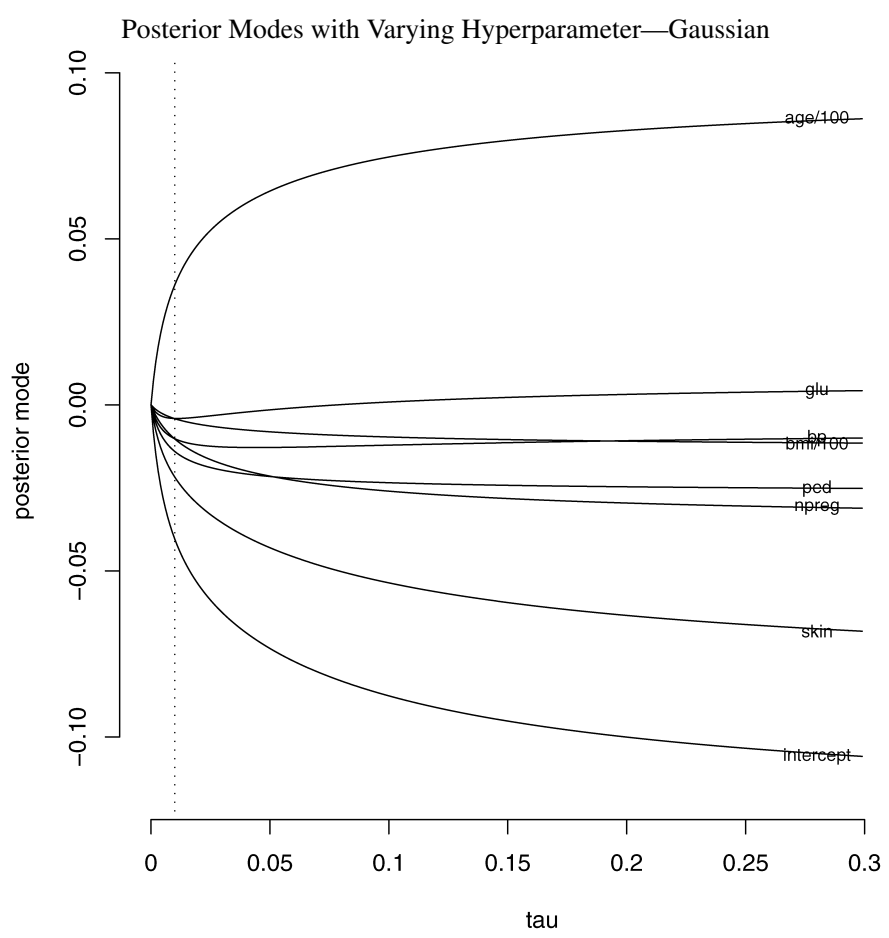

Figure 1. MAP estimates of logistic regression parameters for Gaussian priors with specified variances. The vertical dotted line shows estimates with mean 0 , variance .01 , and Gaussian prior on each parameter.

models. The lasso estimate was defined as a least squares estimate subject to a constraint on the sum of absolute values of the coefficients. Tibshirani observed that this was equivalent to a Bayesian MAP estimate using a Laplace prior, as

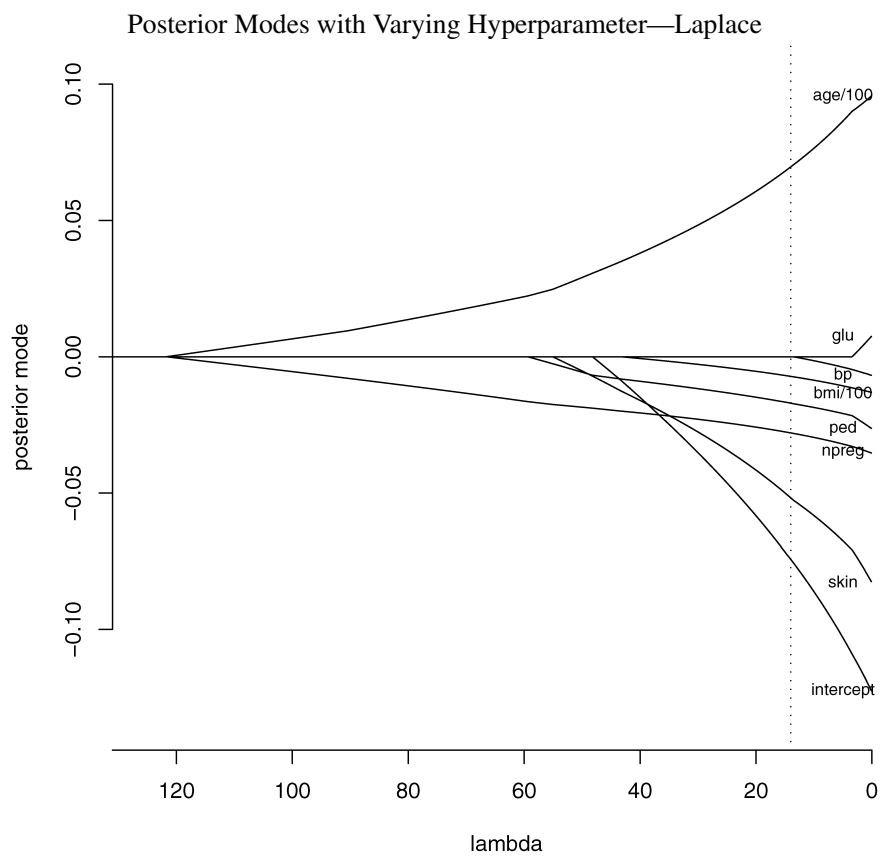

Figure 2. MAP estimates of logistic regression parameters for Laplace priors with specified variances. The data set is the same as in Figure 1 . The vertical dotted line shows estimates with mean 0 , variance .27, Laplace prior on each parameter. 
presented earlier. Since then, the use of constraints or penalties based on the absolute values of coefficients has been used to achieve sparseness in logistic regression and many other data-fitting tasks (Girosi 1998; Tipping 2001; Figueiredo and Jain 2001; Figueiredo 2003; Efron, Hastie, Johnstone, and Tibshirani 2004; Madigan and Ridgeway 2004). Zou and Hastie (2005) discussed some of the limitations of the lasso and a particular generalization.

\section{FINDING THE MAP ESTIMATE}

Ideally we would use the posterior distribution of $\boldsymbol{\beta}$ to compute a posterior predictive distribution for a desired $y_{\text {new }}$, given the corresponding $\mathbf{x}_{\text {new }}$ (Mallick, Ghosh, and Ghosh 2005). In many practical tasks, however, efficiency requires that we base predictions on a point estimate of $\boldsymbol{\beta}$. This simplification does not necessarily lead to less-accurate predictions (Smith 1999).

For the logistic regression model, with the priors that we have discussed, no inexpensive computational procedure for finding the posterior mean seems to exist. Hence we focus on posterior mode estimation. $D$ is

The posterior density for $\boldsymbol{\beta}$ with the logistic link on data set

$$
L(\boldsymbol{\beta})=p(\boldsymbol{\beta} \mid D) \propto\left(\prod_{i=1}^{n} \frac{1}{1+\exp \left(-\boldsymbol{\beta}^{T} \mathbf{x}_{i} y_{i}\right)}\right) p(\boldsymbol{\beta}),
$$

where $p(\boldsymbol{\beta})$ is the prior on $\boldsymbol{\beta}$ and $i$ indexes the training examples in $D$. For Gaussian priors with mean 0 and variance $\tau$ on the $\beta_{j}$ 's, the log posterior (ignoring the normalizing constant) is given by

$$
\begin{aligned}
l(\boldsymbol{\beta})=-\sum_{i=1}^{n} \ln \left(1+\exp \left(-\boldsymbol{\beta}^{T} \mathbf{x}_{i} y_{i}\right)\right) \\
\\
\quad-\sum_{j=1}^{d}\left(\ln \sqrt{\tau}_{j}+\frac{\ln 2 \pi}{2}+\frac{\beta_{j}^{2}}{2 \tau_{j}}\right),
\end{aligned}
$$

and for Laplace priors with mean 0 and variance $2 / \lambda_{j}^{2}$, we have

$$
\begin{aligned}
l(\boldsymbol{\beta})=-\sum_{i=1}^{n} \ln \left(1+\exp \left(-\boldsymbol{\beta}^{T} \mathbf{x}_{i} y_{i}\right)\right) & \\
& -\sum_{j=1}^{d}\left(\ln 2-\ln \lambda_{j}+\lambda_{j}\left|\beta_{j}\right|\right),
\end{aligned}
$$

with $j=1, \ldots, d$ indexing the features in both cases. The MAP estimate is then the $\boldsymbol{\beta}$ that maximizes $l(\boldsymbol{\beta})$ [or minimizes $-l(\boldsymbol{\beta})]$.

\subsection{The Logistic Model From an Optimization Standpoint}

The negated log-posterior for a logistic regression model is convex with either the Gaussian or Laplace prior, and a wide variety of convex optimization algorithms are applicable. For maximum likelihood logistic regression, the most common optimization approach in statistical software is some variant of the multidimensional Newton-Raphson method implemented through iteratively reweighted least squares (Dennis and Schnabel 1989; Hastie and Pregibon 1992). Newton algorithms have the advantage of converging in very few iterations. Furthermove, the matrix of second derivatives that they compute has other uses, for example, in finding asymptotic confidence intervals for parameters.

For high-dimensional problems such as text categorization, however, Newton algorithms have the serious disadvantage of requiring $O\left(d^{2}\right)$ memory, where $d$ is the number of model parameters. Consequently, various alternate optimization approaches have been explored for maximum likelihood and MAP logistic regression in the large- $d$ case (Kivinen and Warmuth 2001; Zhang and Oles 2001; Malouf 2002; Jin, Yan, Zhang, and Hauptmann 2003; Komarek and Moore 2003).

\subsection{The CLG Algorithm for Ridge Logistic Regression}

We based our implementation on the CLG algorithm (Zhang and Oles 2001), a cyclic coordinate descent (Luenberger 1984, sec. 7.9) optimization algorithm tuned for fitting a logistic model with a Gaussian prior, due to its efficiency and ease of implementation. In this section we describe the CLG algorithm in detail.

A cyclic coordinate descent algorithm begins by setting all variables to some initial value. It then sets the first variable to a value that minimizes the objective function, holding all other variables constant. This is a one-dimensional optimization problem. The algorithm then finds the minimizing value of a second variable, while holding all other values constant (including the new value of the first variable). Then the third variable is optimized, and so on. When all variables have been traversed, the algorithm returns to the first variable and starts again. Multiple passes are made over the variables until some convergence criterion is met.

When fitting a ridge logistic regression model, the onedimensional problems involve finding $\beta_{j}^{\text {new }}$, the value for the $j$ th parameter that gives the minimum value for $-l(\boldsymbol{\beta})$, assuming that the other $\beta_{j^{\prime}}$ 's are held at their current values. Finding this $\beta_{j}^{\text {new }}$ is equivalent to finding the $z$ that minimizes

$$
g(z)=\left(\sum_{i=1}^{n} f\left(r_{i}+\left(z-\beta_{j}\right) x_{i j} y_{i}\right)\right)+\frac{z^{2}}{2 \tau_{j}},
$$

where the $r_{i}=\boldsymbol{\beta}^{T} \mathbf{x}_{i} y_{i}$ are computed using the current value of $\boldsymbol{\beta}$ and so are treated as constants, $f(r)=\ln (1+\exp (-r))$, and Gaussian penalty terms not involving $z$ are constant and thus omitted.

The $\beta_{j}^{\text {new }}$ that gives the minimum value of $g(\cdot)$ does not have a closed form, so an optimization procedure must be used even for this one-dimensional problem. Zhang and Oles used a method related to the one-dimensional Newton method (Press, Teukolsky, Vetterling, and Flannery 1992).

The classic Newton method would approximate the objective function $g(\cdot)$ by the first three terms of its Taylor series at the current value of the parameter being optimized ( $\beta_{j}$ for us). This approximation is

$$
\begin{aligned}
\tilde{g}(z) & =g\left(\beta_{j}\right)+g^{\prime}\left(\beta_{j}\right)\left(z-\beta_{j}\right)+\frac{1}{2} g^{\prime \prime}\left(\beta_{j}\right)\left(z-\beta_{j}\right)^{2} \\
& \approx g(z),
\end{aligned}
$$


where for a ridge logistic regression model, we have

$$
\begin{aligned}
g^{\prime}\left(\beta_{j}\right) & =\left.\frac{d g(z)}{d z}\right|_{z=\beta_{j}} \\
& =\left(\sum_{i=1}^{n}-x_{i j} y_{i} \frac{1}{1+\exp \left(r_{i}\right)}\right)+\frac{\beta_{j}}{\tau_{j}} .
\end{aligned}
$$

and

$$
g^{\prime \prime}\left(\beta_{j}\right)=\left.\frac{d^{2} g(z)}{d^{2} z}\right|_{z=\beta_{j}}=\left(\sum_{i=1}^{n} x_{i j}^{2} \frac{\exp \left(r_{i}\right)}{\left(1+\exp \left(r_{i}\right)\right)^{2}}\right)+\frac{1}{\tau_{j}} .
$$

The approximation in (11) has its minimum at

$$
\beta_{j}^{(\text {new })}=\arg \min _{z} g(z)=\beta_{j}-\frac{g^{\prime}\left(\beta_{j}\right)}{g^{\prime \prime}\left(\beta_{j}\right)} .
$$

The increment that we must add to $\beta_{j}$ to reach $\beta_{j}^{(\text {new })}$ is then

$$
\Delta \beta_{j}=\beta_{j}^{(\text {new })}-\beta_{j}=-\frac{g^{\prime}\left(\beta_{j}\right)}{g^{\prime \prime}\left(\beta_{j}\right)} .
$$

Note that because $\tilde{g}^{\prime \prime}(z)=g^{\prime \prime}(z)$ is strictly positive (assuming that $x_{i j} \neq 0$ for some $i$ ), we know that we have a minimum.

In CLG, Zhang and Oles modified the update in (15) in three ways. First, as in most applications of Newton's method, convergence requires avoiding large updates in regions where a quadratic is a poor approximation to the objective. Zhang and Oles specified a value $\Delta_{j}>0$ that $\left|\Delta \beta_{j}\right|$ is not allowed to exceed on a single iteration. This is similar to the trust region approach to Newton's method (Dennis and Schnabel 1989). Zhang and Oles presented several alternative update rules for adapting the width, $2 \Delta_{j}$, of the trust region from iteration to iteration. We used the update

$$
\Delta_{j}^{\text {new }}=\max \left(2\left|\Delta \beta_{j}\right|, \Delta_{j} / 2\right),
$$

where $\Delta_{j}$ is the trust region half-width used with $\beta_{j}$ on the current pass through the coordinates, $\Delta \beta_{j}$ is the update made to $\beta_{j}$ on this pass, and $\Delta_{j}^{\text {new }}$ is the trust region half-width to be used on the next pass.

Second, instead of using a truncated Taylor series [eq. (11)] as their quadratic approximation in Newton's method, CLG uses

$$
\begin{aligned}
\hat{g}(z) & =g\left(\beta_{j}\right)+g^{\prime}\left(\beta_{j}\right)\left(z-\beta_{j}\right)+\frac{1}{2} G\left(\beta_{j}\right)\left(z-\beta_{j}\right)^{2} \\
& \approx g(z),
\end{aligned}
$$

where

$$
G\left(\beta_{j}\right)=\left(\sum_{i=1}^{n} x_{i j}^{2} F\left(r_{i}, \Delta_{j}\left|x_{i j}\right|\right)\right)+\frac{1}{\tau_{j}} .
$$

Zhang and Oles allow $F(r, \delta)$ to be any convenient function that satisfies (for $\delta>0$ )

$$
\begin{aligned}
F(r, \delta) & \geq \sup _{|\Delta r| \leq \delta} f^{\prime \prime}(r+\Delta r) \\
& =\sup _{|\Delta r| \leq \delta} \frac{\exp (r+\Delta r)}{(1+\exp (r+\Delta r))^{2}} .
\end{aligned}
$$

In words, $F\left(r_{i}, \Delta_{j}\left|x_{i j}\right|\right)$ is an upper bound on the second derivative of $f$ for values of $r_{i}$ reachable by updates in the trust region. For the logistic model, Zhang and Oles used

$F(r, \delta)=\min \left(.25, \frac{1}{2 \exp (-\delta)+\exp (r-\delta)+\exp (-r-\delta)}\right)$

In our implementation, we used

$$
F(r, \delta)= \begin{cases}.25, & \text { if }|r| \leq \delta \\ \frac{1}{2+\exp (|r|-\delta)+\exp (\delta-|r|)}, & \text { otherwise }\end{cases}
$$

Using $\hat{g}(\cdot),(15)$ gives the following update for a Gaussian prior:

$$
\begin{aligned}
\Delta v_{j} & =-\frac{\hat{g}^{\prime}\left(\beta_{j}\right)}{\hat{g}^{\prime \prime}\left(\beta_{j}\right)} \\
& =\frac{\sum_{i=1}^{n}\left(x_{i j} y_{i}\right) /\left(1+\exp \left(r_{i}\right)\right)-\beta_{j} / \tau_{j}}{\sum_{i=1}^{n} x_{i j}^{2} F\left(r_{i}, \Delta_{j}\left|x_{i j}\right|\right)+1 / \tau_{j}} .
\end{aligned}
$$

Applying the trust region restriction then gives the actual update used in CLG,

$$
\Delta \beta_{j}= \begin{cases}-\Delta_{j} & \text { if } \Delta v_{j}<-\Delta_{j} \\ \Delta v_{j} & \text { if }-\Delta_{j} \leq \Delta v_{j} \leq \Delta_{j} \\ \Delta_{j} & \text { if } \Delta_{j}<\Delta v_{j}\end{cases}
$$

Third, instead of iterating $\beta_{j}^{(\text {new })}=\beta_{j}+\Delta \beta_{j}$ to convergence, CLG does this only once before going on to the next $\beta_{j^{\prime}}$. The optimal value of $\beta_{j}^{(\text {new })}$ during a particular pass through the coordinates depends on the current values of the other $\beta_{j}$ 's. These are themselves changing, so there is little reason to tune $\beta_{j}^{(\text {new })}$ to high precision. We simply want to decrease $g(\cdot)$, and thus decrease $-l(\boldsymbol{\beta})$, before going on to the next $\beta_{j^{\prime}}$.

Summarizing, Figure 3 presents pseudocode for our implementation of CLG. Zhang and Oles presented several possibilities for the convergence test. Our code declares convergence when $\left(\sum_{i=1}^{n}\left|\Delta r_{i}\right|\right) /\left(1+\sum_{i=1}^{n}\left|r_{i}\right|\right) \leq \epsilon$, where $\sum_{i=1}^{n}\left|\Delta r_{i}\right|$ is sum of the changes in the linear scores of the training examples between the beginning and end of a pass through the coordinates, and $\epsilon$ is a user specified tolerance. The optimization is considered to converge if the change is small either in absolute terms or as a fraction of the magnitude of the linear scores. The experiments that we report here use $\epsilon=.0005$, but larger values can be used with only a minimal impact on effectiveness.

Some implementation details are worth noting. Like Zhang and Oles, we maintain for each training document the value, $r_{i}$, of the dot product between the current $\boldsymbol{\beta}$ and $\mathbf{x}_{i}$. Each time that a parameter $\beta_{j}$ in $\boldsymbol{\beta}$ is updated, the affected values of $r_{i}$ are updated. Only the $r_{i}$ 's for examples in which $x_{i j} \neq 0$ are affected by a change in $\beta_{j}$, and because text data are sparse, the vast majority of the $x_{i j}$ 's are equal to 0 . Therefore, we can update the $r_{i}$ 's very efficiently by storing the vectors in inverted index form, that is, as an array of triples $\left(j, i, x_{i j}\right)$, sorted by $j$, containing entries for all and only the nonzero values of $x_{i j}$. 
Algorithm 1: CLG (Zhang and Oles 2001)

(1) initialize $\beta_{j} \leftarrow 0, \Delta_{j} \leftarrow 1$ for $j=1, \ldots, d ; r_{i} \leftarrow 0$ for $i=1, \ldots, n$

(2) for $k=1,2, \ldots$ until convergence

(3) for $j=1, \ldots, d$

compute tentative step $\Delta v_{j} \quad$ [eq. 18]

$\Delta \beta_{j} \leftarrow \min \left(\max \left(\Delta v_{j},-\Delta_{j}\right), \Delta_{j}\right) \quad$ (limit step to trust region)

$\Delta r_{i} \leftarrow \Delta \beta_{j} x_{i j} y_{i}, r_{i} \leftarrow r_{i}+\Delta r_{i}$ for $i=1, \ldots, n$

$\beta_{j} \leftarrow \beta_{j}+\Delta \beta_{j}$

end

$\Delta_{j} \leftarrow \max \left(2\left|\Delta \beta_{j}\right|, \Delta_{j} / 2\right) \quad$ (update size of trust region)

end

Figure 3. The Zhang and Oles CLG algorithm with our choice of trust region update.

\subsection{Modifying CLG for the Laplace Prior}

We modified the CLG algorithm to fit lasso logistic regression models as well. Because the log-posterior density corresponding to a Laplace prior lacks finite first and second derivatives when one or more $\beta_{j}$ 's are 0 , some special handling is necessary.

With the Laplace prior, the Zhang-Oles tentative update is defined only for $\beta_{j} \neq 0$ and is

$$
\begin{aligned}
\Delta v_{j} & =-\frac{\hat{g}^{\prime}\left(\beta_{j}\right)}{\hat{g}^{\prime \prime}\left(\beta_{j}\right)} \\
& =\frac{\sum_{i=1}^{n} x_{i j} y_{i} /\left(1+\exp \left(r_{i}\right)\right)-\lambda_{j} \operatorname{sgn}\left(\beta_{j}\right)}{\sum_{i=1}^{n} x_{i j}^{2} F\left(r_{i}, \Delta_{j}\left|x_{i j}\right|\right)},
\end{aligned}
$$

where $\operatorname{sgn}\left(\beta_{j}\right)$ is +1 for $\beta_{j}>0$ and -1 for $\beta_{j}<0$. This update has two problems: It is undefined at $\beta_{j}=0$, and it is not guaranteed to decrease the objective if it would change the sign of $\beta_{j}$. [The necessary condition on $F(\cdot)$ does not hold for intervals that span 0.]

We solve the second problem simply by setting $\beta_{j}^{(\text {new })}$ to 0 if the update would otherwise change its sign. To deal with the first problem, when the starting value of $\beta_{j}$ is 0 , we attempt an update in both directions and see whether either one succeeds. That is, if setting $\operatorname{sgn}\left(\beta_{j}\right)$ to +1 in (20) yields a $\Delta v_{j}>0$, or setting $\operatorname{sgn}\left(\beta_{j}\right)$ to -1 yields a $\Delta v_{j}<0$, we then accept the corresponding update; otherwise, we keep $\beta_{j}$ at 0 . Note that at most one update direction can be successful, due to the convexity of $g(\cdot)$. The resulting $C L G$-lasso algorithm is identical to the CLG (Fig. 3), except that the computation of $\Delta v_{j}$ is done as shown in Figure 4.

\subsection{Selecting the Hyperparameter}

The Gaussian and Laplace priors both require a prior variance, $\sigma_{j}^{2}$, for parameter values. (The actual hyperparameters are $\tau_{j}=\sigma_{j}^{2}$ for the Gaussian and $\lambda_{j}=\sqrt{2} / \sigma_{j}$ for the Laplace.) We tried two approaches to setting the prior variance. The first was simply

$$
\sigma_{j}^{2}=\frac{d}{u}=d n / \sum_{i=1}^{n}\left\|\mathbf{x}_{i}\right\|_{2}^{2},
$$

where $d$ is the number of predictor features (plus 1 for the constant term) and $u$ is the mean squared Euclidean norm of the training examples (after feature selection, if any). This heuristic was loosely inspired by a similar heuristic used to choose the regularization parameter in SVM_Light (Sec. 5.3.1). We call the value chosen in this way the norm-based value of the hyperparameter.

We also tested choosing the hyperparameter by partial 10fold cross-validation on the training set. For each category, we randomly separated the training set into 10 portions and did 2 runs of training on 9 portions and testing on the 10th (validation) portion. (Preliminary experiments showed that using two folds gave results very similar to those using all 10 folds, and was of course 5 times faster.) In each run we tested values for the Laplace hyperparameter $\lambda_{j}$ from the range $0.01-316$ by multiples of $\sqrt{10}$, or values for the Gaussian hyperparameter $\tau_{j}$ from the range $.0001-10,000$ by multiples of 10 . For each choice of the hyperparameter, we computed the sum of loglikelihoods for the documents in the validation portions and chose the hyperparameter value that maximized this sum. We call this the cross-validated value of the hyperparameter.

\subsection{Implementation}

We have publicly released an open-source $\mathrm{C}++$ implementation of the foregoing algorithms, Bayesian binary re-
Algorithm 2: Computation of $\Delta v_{j}$ in CLG-lasso$$
\text { if } \beta_{j}=0
$$$$
s \leftarrow 1 \text { (try positive direction) }
$$$$
\text { compute } \Delta v_{j} \text { by formula (20) }
$$$$
\text { if } \Delta v_{j} \leq 0 \quad \text { (positive direction failed) }
$$$$
s \leftarrow-1 \quad \text { (try negative direction) }
$$$$
\text { compute } \Delta v_{j} \text { by formula (20) }
$$$$
\text { if } \Delta v_{j} \geq 0 \quad \text { (negative direction failed) }
$$$$
\Delta v_{j} \leftarrow 0
$$$$
\text { endif }
$$$$
\text { endif }
$$
else

$$
s \leftarrow \beta_{j} /\left|\beta_{j}\right|
$$
compute $\Delta v_{j}$ by formula (20)$$
\text { if } \left.s\left(\beta_{j}+\Delta v_{j}\right)<0 \quad \text { (cross over } 0\right)
$$$$
\text { endif }
$$$$
\Delta v_{j} \leftarrow-\beta_{j}
$$
endif

Figure 4. Computation of $\Delta v_{j}$ in the CLG-lasso algorithm. 
gression (BBR), at http://bayesianregression.org, and have used it in a wide variety of applications. We have also developed an extension of the algorithms to polytomous logistic regression (Madigan et al. 2005a,b) and provide an implementation, Bayesian multinomial regression (BMR), at http://bayesianregression.org. Both BBR and BMR include an "autosearch" capability for hyperparameter selection. Similar algorithms for logistic regression have been developed by Shevade and Keerthi (2003), Krishnapuram, Hartemink, Carin, and Figueiredo (2005), and others.

\section{METHODS}

We tested lasso logistic regression on five text categorization data sets. In this section we discuss how texts were represented as numeric vectors, what documents and category labels were used, and how effectiveness was measured and tuned. We also discuss two state-of-the-art text categorization approaches used as benchmarks: support vector machines (SVMs), and ridge logistic regression combined with feature selection.

\subsection{Text Representation}

Representing a document for statistical classification has two major aspects: text processing and term weighting. Text processing breaks the character string into tokens, that is, individual terms, such as words or phrases. We used very simple methods, as described later. Term-weighting methods tabulate the number of occurrences of each distinct term in a document and across documents. They then compute a numeric weight for each term with respect to each document. We represent each document as a vector of such weights.

We used a form of $T F \times I D F$ (term frequency times inverse document frequency) term weighting with cosine normalization (Salton and Buckley 1988). This gives term $j$ in document $i$ an initial unnormalized weight of

$$
x_{i j}^{u}= \begin{cases}0 & \text { if } n(i, j)=0 \\ (1+\ln n(i, j)) \ln \frac{|\mathcal{D}|+1}{n(j)+1}, & \text { otherwise, }\end{cases}
$$

where $n(j)$ is the number of training documents that contain term $j, n(i, j)$ is the number of occurrences of term $j$ in document $i$, and $|\mathcal{D}|$ is the total number of training documents. Incrementing the IDF numerator and denominator by 1 is a variant of IDF weighting that gives terms occurring only on the test set a defined IDF value. All experiments separate the data into training and test sets, and all IDF weights are based on the training set.

Then, to reduce the impact of document length, we "cosinenormalize" the feature vectors to have a Euclidean norm of 1.0. The final weights are

$$
x_{i j}=\frac{x_{i j}^{u}}{\sqrt{\sum_{j^{\prime}} x_{i j^{\prime}}^{u} \times x_{i j^{\prime}}^{u}}} .
$$

The summation is over all terms in the corpus, but, of course, most $x_{i j}=0$.

In addition to one parameter for each term, our vectors include a constant term, 1.0 (which is omitted from cosine normalization). A constant term is usual in statistical practice but is often omitted in text categorization studies. The experiments here use a Gaussian or Laplace prior on the model parameter for the constant term, just as for the other model parameters.

\subsection{Data Sets}

Our experiments used five standard text categorization test collections. Three of these-ModApte, RCV1-v2, and OHSUMED — each contain on the order of 100 distinct binary classification problems corresponding to predicting expert human indexing decisions. The other two-WebKB Universities and 20 Newsgroups-are based on a smaller number of binary classifications of uncertain quality but are included because they are widely used in published research.

5.2.1 ModApte. Our first collection was the ModApte subset of the Reuters-21578 collection of news stories (Lewis 2004) available at (http://www.daviddlewis.com/resources/ testcollections/reuters21578/). The ModApte subset contains 9,603 training documents and 3,299 test documents. Documents in the ModApte data set belong to 0 or more of a set of "topic" categories corresponding to news areas of economic interest. We used the 90 topic categories that have at least 1 positive training example and 1 positive test example on the ModApte subset.

Text processing used Lemur (see http://www-2.cs.cти.edu/ lemur/), which performed a simple tokenization into words (using its TrecParser module), discarded words from the SMART stopword list of 572 words (available at ftp://ftp.cs.cornell.edu/ pub/smart/english.stop or as part of the RCV1-v2 data set), and applied the Lemur variant of the Porter stemmer (Porter $1980,2003)$ to remove word endings. All stems from text in the $<$ TITLE $>$ and $<$ BODY $>$ SGML elements were combined to produce raw TF weights. There were 21,989 unique terms in the ModApte data set, 18,978 of which occured in the training set and thus potentially had nonzero parameters in a classifier.

5.2.2 RCV1-v2. The second data set was RCV1-v2, a test categorization test collection of 804,414 newswire stories based on data released by Reuters, Ltd. (available at $h t t p: / / t r e c$. nist.gov/data/reuters/reuters.html). We used the LYRL2004 training/test split (Lewis et al. 2004) of RCV1-v2, which contains 23,149 training documents and 781,265 test documents. However, for efficiency we took a fixed, random, roughly $10 \%$ subset (77,993 documents) of the test documents as our test set in all experiments.

We used all 103 RCV1-v2 "topic" categories in our experiments. The topic categories in RCV1-v2 are different from those in Reuters-21578 and cover a broader range of news types. Two of the categories have no positive training examples, and so a default classifier that predicts "no" for all test documents was assumed. Classifiers were trained for the other 101 categories. Each RCV1-v2 document is known to belong to at least 1 of the remaining 101 categories, but we did not use that fact in classification.

Term weights were computed from stemmed token files distributed with RCV1-v2. A total of 47,152 unique terms were present in the training set, and 288,062 unique terms were present in the union of the training set and the 77,993-document test set.

5.2.3 OHSUMED. The third data set consists of Medline records from 1987-1991, formatted for the SMART retrieval system and distributed as part of the OHSUMED textretrieval test collection (Hersh, Buckley, Leone, and Hickman 1994) (available at ftp://medir.ohsu.edu/pub/ohsumed/). Of the 
348,566 OHSUMED records, we used the 233,445 records in which the title, abstract, and medical subject headings (MeSH) category fields were all nonempty. We used the 83,944 such documents from 1987 and 1988 as our training set and the 149,501 such documents from 1989-1991 as our test set. Our binary classification tasks were to predict the presence or absence of MeSH (Lowe and Barnett 1994) controlled vocabulary categories in the records. We used the same 77 categories as used by Lewis, Schapire, Callan, and Papka (1996).

The text processing was the same as for ModApte. All text from the.$T$ (title) and.$W$ (abstract) fields was used in computing TF weights. There were 73,269 distinct terms in the training set and 122,076 terms in the union of the training and test sets.

5.2.4 WebKB Universities. This data set (http://www.cs. cтu.edu/afs/cs.cmu.edu/project/theo-20/www/data/) contains HTML pages downloaded from university computer science departments in January 1997. The data set comprises 8,282 pages, each of which is manually classified into exactly 1 of 7 categories: student, faculty, staff, department, course, project, or other. However, we treated these as seven binary classification problems. Four universities are represented by a large number of pages: Cornell (867 pages), Texas (827), Washington $(1,205)$, and Wisconsin $(1,263)$. Another 4,120 miscellaneous pages were collected from a range of other universities.

We followed the methodological recommendations from the foregoing web page. The Rainbow system (available at http://www.cs.cmu.edu/mccallum/bow) was used to tokenize the documents. Training was performed on documents from three of the main universities plus those from miscellaneous universities, with testing on the pages from the fourth, held-out university. This was repeated, using each of the four main universities as a test set once, and the results were averaged. Thus number of terms in the training set varied, ranging up to 85,600.

5.2.5 20 Newsgroups. The final test collection, 20 Newsgroups, consists of roughly equal-sized samples of postings to 20 Usenet newsgroups. Some postings belong to more than 1 newsgroup, and we treated the data set as specifying 20 binary classification problems. We used the "by date" training/test split (see http://people.csail.mit.edu/people/jrennie/20Newsgroups/) giving 11,314 training documents and 7,532 test documents.

After discarding all header fields except Subject:, text processing was the same as for ModApte. There were 102,760 distinct terms in the training set, and 138,700 terms in the union of the training and test sets.

\subsection{Benchmark Algorithms}

We compared lasso logistic regression to two alternatives.

5.3.1 Support Vector Machines. The SVM algorithm for learning linear classifiers is consistently one of the most effective approaches to text categorization (Joachims 1998; Zhang and Oles 2001; Lewis et al. 2004). It produces models with a form of dual-space sparseness that may or may not translate into sparseness of linear model coefficients.

We trained a single SVM classifier for each category using version 5.00 of SVM_Light (Joachims 1998, 2002) (available at $h t t p: / / s v m l i g h t$.joachims.org/). All software parameters were left at default values except the regularization parameter C (option $-c$ ). The value of $C$ was selected from the range $10^{-4}-10^{4}$ (by multiples of 10) using 10-fold cross-validation. The $\mathrm{C}$ that gave the highest cross-validated estimate for the sum of hinge losses $\left[\sum_{i=1}^{n} \max \left(0,-\boldsymbol{\beta}^{T} \mathbf{x}_{i} y_{i}\right)\right]$ was chosen. We found this to be more effective than the usual approach of maximizing crossvalidated classification accuracy.

5.3.2 Ridge Logistic Regression With Feature Selection. Ridge logistic regression is widely used in text classification, but usually only after low-quality features are discarded. We tested three feature selection methods in combination with ridge logistic regression, and also evaluated no feature selection.

The feature selection approaches each computed some quality measure for each feature, ranked features by that measure, and then used only the top-ranked features when learning a classifier. A different set of features was chosen for each category. We tested each method of choosing feature sets with 5, 50, or 500 features, with the same number of features used for all categories. The constant term was always used, so the total number of model parameters was 6,51 , and 501. We did not test more computationally expensive approaches to choosing the number of features, such as cross-validation.

The first feature quality measure was the chi-squared test for independence between two variables. This measure chooses the features that are least independent from the class label and is widely used in text categorization (Yang and Pedersen 1997; Sebastiani 2002). The chi-squared measure is based on a $2 \times 2$ contingency table between a predictor term $j$ and a predicted category label. Let $a$ be the number of training documents in the category and containing term $j$ ("true positives"), let $b$ be the number in the category but not containing $j$ ("false negatives"), let $c$ be the number not in the category but containing $j$ ("false positives"), and let $d$ be the number neither in the category nor containing $j$ ("true negatives"). Let $n=a+b+c+d$ be the total number of training documents. Then the chi-squared measure is

$$
\chi^{2}=\frac{n(a d-b c)^{2}}{(a+b)(c+d)(a+c)(b+d)} .
$$

Our second measure, binormal separation (BNS), was the best measure in a recent study of feature selection for text categorization (Forman 2003). Forman defined BNS as

$$
B(j)=\left|\Phi^{-1}\left(\frac{a}{a+b}\right)-\Phi^{-1}\left(\frac{c}{c+d}\right)\right|,
$$

where $\Phi$ is the standard normal cumulative distribution function. We used Ackham's algorithm to compute $\Phi^{-1}$ (Ackham 2004) and, as suggested by Forman, replaced values of $\Phi^{-1}(0)$ by .0005 . Forman justified BNS in terms of receiver operating characteristic analysis.

Most feature selection measures used in text classification (including the two just discussed) take into account only the presence or absence of terms in documents. Our third measure - the Pearson product-moment correlation-makes use of term weights in choosing features. This measure is

$$
r_{j}=\frac{\sum_{i=1}^{n}\left(x_{i j}-\overline{x^{(j)}}\right)\left(y_{i}-\bar{y}\right)}{\sqrt{\sum_{i=1}^{n}\left(x_{i j}-\overline{x^{(j)}}\right)^{2}} \sqrt{\sum_{i=1}^{n}\left(y_{i}-\bar{y}\right)^{2}}},
$$

where $\overline{x^{(j)}}$ is the mean of $x_{i j}$ across the training documents and $\bar{y}$ is the mean of $y_{i}(+1$ or -1 class labels) for the category of 
interest across the training documents. We use $\left|r_{j}\right|$ as our feature quality measure. The Pearson product-moment correlation measures the degree to which there is a linear relationship between the $x_{i j}$ 's for some term $j$ and the $y_{i}$ 's for the category of interest. It has been used for feature selection in various machine learning tasks.

\subsection{Effectiveness Measure}

One effectiveness measure is the number of test set errors (false negatives + false positives) or, if normalized, the error rate,

$$
E=\frac{\text { false positives }+ \text { false negatives }}{|\mathcal{T}|},
$$

where $|\mathcal{T}|$ is the size of the test set. We also measured effectiveness of our classifiers using van Rijsbergen's $F$ measure (van Rijsbergen 1972, 1979; Lewis 1995). We set the $F$ measure's parameter $\beta$ (not to be confused with the $\beta$ 's of our logistic models) to 1.0 , giving the measure $F 1$ :

$$
F 1=\frac{2 \times \text { true positives }}{2 \times \text { true positives }+ \text { false positives }+ \text { false negatives }} .
$$

We define $F 1$ to have the value 1.0 if the denominator is 0 , which can occur if no test set documents belong to the class of interest. We sometimes report the unweighted arithmetic mean of $F 1$ across the categories for a particular test collection, that is, the macroaveraged $F 1$.

\subsection{Threshold Selection}

Logistic regression models produce an estimate of $p(y=$ $\left.+1 \mid \boldsymbol{\beta}, \mathbf{x}_{i}\right)$, the probability that vector $\mathbf{x}_{i}$ belongs to the category of interest. For classification, we must convert these estimates to binary class label predictions. The simplest approach is to predict $y=+1$ (assign the category) when $p\left(y=+1 \mid \boldsymbol{\beta}, \mathbf{x}_{i}\right) \geq .5$. This minimizes the expected test set error rate when the predicted probabilities equal the actual probabilities used in a probabilistic labeling. We refer to .5 as the default threshold.

Model inadequacies and finite training data mean that the probabilities produced by the logistic regression models may not be well calibrated. Thus we also tested setting the threshold for each category to the highest value that gave the minimum possible number of training set classification errors. We call these the tuned thresholds.
Maximizing $F 1$ is trickier than minimizing the error rate, because the optimal expected value of $F 1$ cannot be achieved by any single preset threshold for all test sets (Lewis 1995). For simplicity, we used the same thresholds when evaluating by $F 1$ that we used when evaluating by error rate. For linear models produced by SVM_Light we tried both a default threshold (0), and a tuned threshold chosen by minimizing number of training set errors.

\section{RESULTS}

We evaluated both the raw effectiveness of lasso logistic regression for text categorization and this regression's ability to provide more compact classifiers than competing approaches.

\subsection{Effectiveness of Lasso Logistic Regression}

Our first set of experiments compared the effectiveness of lasso logistic regression with ridge logistic regression and SVMs. Each algorithm used all training set features and a regularization parameter tuned by cross-validation on the training set. Table 1 compares macroaveraged F1 values for the three algorithms (with both default and tuned thresholds) on the five test collections. Lasso logistic regression outperforms ridge logistic regression under all conditions. With default thresholds, it outperforms SVMs on four of five data sets, and with tuned thresholds it outperforms SVMs on three of five data sets.

To test the significance of differences among the algorithms, we looked at the difference in per-category $F 1$ values between pairs of algorithms and applied the two-tailed Wilcoxon matched-pairs signed-ranks test (Table 2). For all categories, we calculated the differences in $F 1$ values and ranked them from smallest to largest by absolute value. We then compared the sum of positive ranks (first method has greater $F 1$ ) and the sum of negative ranks (second method has greater $F 1$ ). Algorithms significantly better at the $p=.05$ level are indicated in bold. By this measure, lasso logistic regression was significantly better than ridge logistic regression on ModApte, RCV1-v2, and OHSUMED and similar to ridge logistic regression on WebKB and 20 Newsgroups. Lasso logistic regression was significantly better than SVMs on WebKB, significantlyworse on 20 Newsgroups, and tied on the other data sets. Note that these significance tests require a debatable assumption of independence among categories.

Table 1. Text categorization effectiveness (macroaveraged F1) on five collections

\begin{tabular}{lcccccc}
\hline \hline & & \multicolumn{5}{c}{ Test collection } \\
\cline { 3 - 7 } Algorithm & Threshold & ModApte & RCV1-v2 & OHSUMED & WebKB & 20 NG \\
\hline Lasso & Default & 48.64 & 54.75 & 47.23 & 46.96 & 73.24 \\
Ridge & Default & 37.63 & 47.72 & 36.09 & 45.73 & 71.47 \\
SVM & Default & 37.69 & 44.87 & 25.28 & 44.14 & 75.15 \\
& & & & & & \\
Lasso & Tuned & 52.03 & 57.66 & 51.30 & 47.28 & 75.13 \\
Ridge & Tuned & 39.71 & 52.42 & 42.99 & 46.03 & 73.67 \\
SVM & Tuned & 52.09 & 57.26 & 49.35 & 39.50 & 81.19 \\
\hline
\end{tabular}


Table 2. Two-tailed Wilcoxon paired signed-ranks test on per-category $F$-measure values

\begin{tabular}{llrrr}
\hline \hline & & \multicolumn{2}{c}{ Sum of ranks } & \\
\cline { 3 - 4 } Collection & Algorithms & Positive & Negative & $p$ value \\
\hline ModApte & Lasso-ridge & $1,667.0$ & 163.0 & .000 \\
$\quad$ (90 categories) & Lasso-SVM & 969.5 & 921.5 & .863 \\
& Ridge-SVM & 204.5 & $1,811.5$ & .000 \\
RCV1-v2 & Lasso-ridge & $3,925.0$ & 926.0 & .000 \\
(103 categories) & Lasso-SVM & $2,112.0$ & $2,739.0$ & .267 \\
& Ridge-SVM & $1,031.0$ & $3,625.0$ & .000 \\
OHSUMED & Lasso-ridge & $2,359.0$ & 416.0 & .000 \\
(77 categories) & Lasso-SVM & $1,462.0$ & $1,239.0$ & .540 \\
& Ridge-SVM & 599.0 & $2,102.0$ & .000 \\
WebKB & Lasso-ridge & 197.0 & 128.0 & .353 \\
(7 categories) & Lasso-SVM & 299.0 & 79.0 & .008 \\
& Ridge-SVM & 228.0 & 123.0 & .182 \\
20 NG & Lasso-ridge & 156.0 & 54.0 & .057 \\
$(20$ categories) & Lasso-SVM & 1.0 & 209.0 & .000 \\
& Ridge-SVM & 0.0 & 210.0 & .000 \\
\hline
\end{tabular}

NOTE: The number of categories in each test collection is shown in first column.

Figure 5 is a parallel-coordinates plot showing the number of test set errors for each algorithm on the three data sets with largest number of categories. Each connected triple of points shows the number of test set errors made by ridge, lasso, and SVM for a particular category. This plot serves as a reminder that for any given category, most test examples are clear negatives and are classified similarly by all three algorithms.
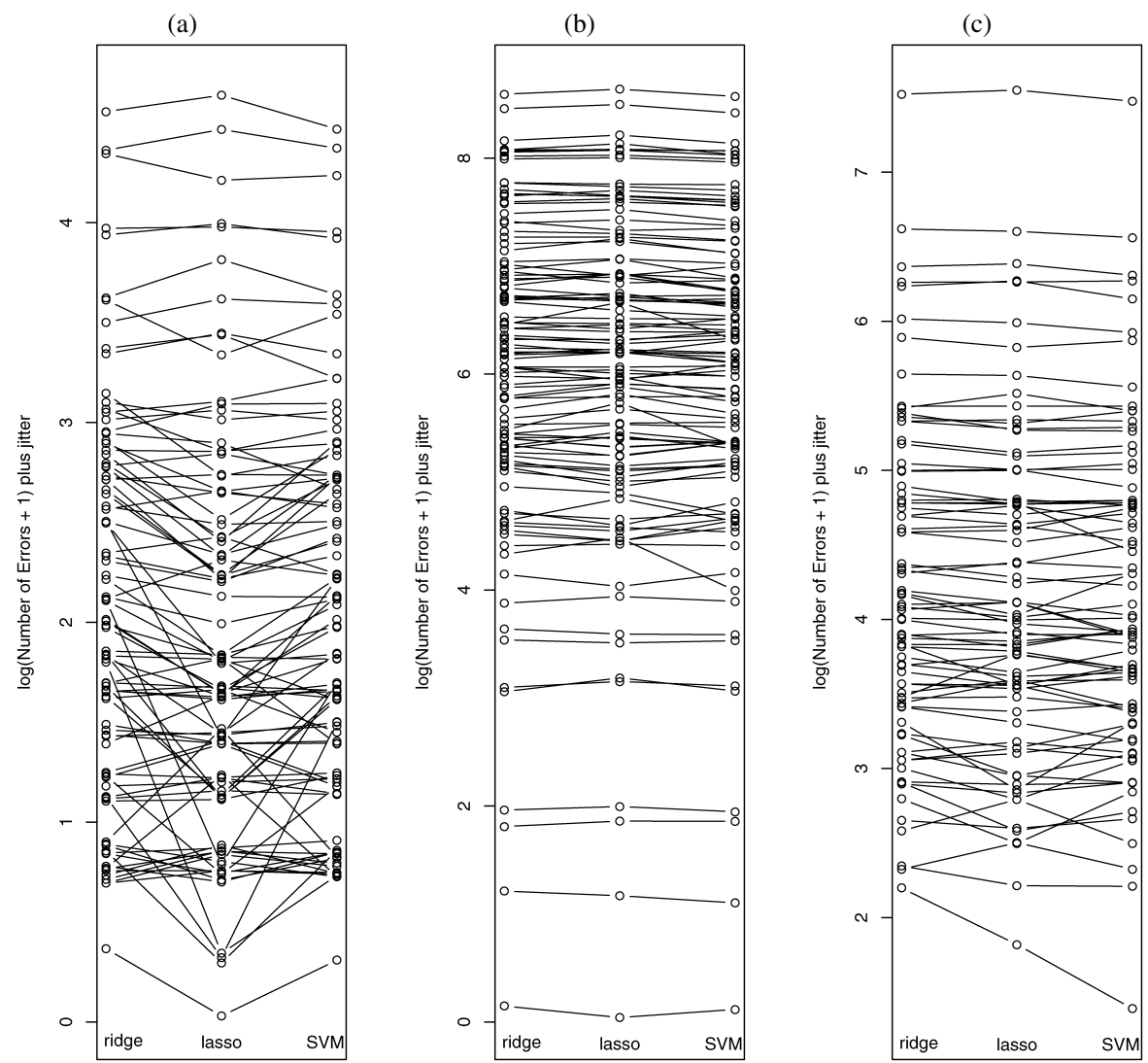

Figure 5. The number of test set errors for each category. (a) ModApte (90 categories); (b) RCV1-v2 (103 categories); (c) OHSUMED (77 categories). Results are shown for ridge logistic regression, lasso logistic regression, and SVMs on the three test collections with the largest number of categories. The vertical axes show the logarithm of 1 plus the number of test set errors, plus a small uniform random jitter to separate the categories. All algorithms use default thresholds, a regularization parameter chosen by training set cross-validation, and no feature selection. 
Table 3. Text categorization effectiveness (macroaveraged F1) on subsets of features

\begin{tabular}{|c|c|c|c|c|}
\hline \multirow[b]{2}{*}{ Collection } & \multirow[b]{2}{*}{ Algorithm } & \multirow[b]{2}{*}{ Number of features } & \multicolumn{2}{|c|}{ Hyperparameter } \\
\hline & & & $\mathrm{CV}$ & Norm \\
\hline \multirow[t]{5}{*}{ ModApte } & Lasso & All $(18,978)$ & 52.03 & 52.58 \\
\hline & Ridge & All $(18,978)$ & 39.71 & 42.17 \\
\hline & Ridge & 500 & 50.14 & 41.40 \\
\hline & Ridge & 50 & 51.72 & 49.14 \\
\hline & Ridge & 5 & 47.04 & 49.48 \\
\hline \multirow{5}{*}{ RCV1-v2 } & Lasso & All $(47,152)$ & 57.66 & 56.56 \\
\hline & Ridge & All $(47,152)$ & 52.42 & 52.85 \\
\hline & Ridge & 500 & 51.90 & 50.82 \\
\hline & Ridge & 50 & 44.84 & 46.31 \\
\hline & Ridge & 5 & 31.21 & 32.54 \\
\hline \multirow[t]{5}{*}{ OHSUMED } & Lasso & All $(122,076)$ & 51.30 & 48.92 \\
\hline & Ridge & All $(122,076)$ & 42.99 & 42.74 \\
\hline & Ridge & 500 & 43.46 & 40.24 \\
\hline & Ridge & 50 & 44.27 & 45.64 \\
\hline & Ridge & 5 & 42.40 & 41.98 \\
\hline \multirow[t]{5}{*}{ WebKB } & Lasso & All (varies) & 47.16 & 44.94 \\
\hline & Ridge & All (varies) & 46.07 & 42.50 \\
\hline & Ridge & 500 & 45.11 & 42.30 \\
\hline & Ridge & 50 & 36.62 & 36.60 \\
\hline & Ridge & 5 & 24.58 & 24.69 \\
\hline \multirow[t]{5}{*}{$20 \mathrm{NG}$} & Lasso & All $(102,760)$ & 75.13 & 76.31 \\
\hline & Ridge & All $(102,760)$ & 73.67 & 76.44 \\
\hline & Ridge & 500 & 74.63 & 73.44 \\
\hline & Ridge & 50 & 66.65 & 66.94 \\
\hline & Ridge & 5 & 53.87 & 53.97 \\
\hline
\end{tabular}

NOTE: Results are for lasso and ridge logistic regression with prior variances chosen using training set crossvalidation $(\mathrm{CV})$ or the norm-based heuristic (norm). Default thresholds were used. Feature subsets of the specified size were chosen for each category using the BNS criterion.

\subsection{Comparing Lasso With Feature Selection}

In text categorization, ridge logistic regression is often used in combination with feature selection,producing sparser and more effective classifiers. To test this approach, we chose feature sets of size 5, 50, and 500 features for each category using each of our three feature selection methods. We fit ridge logistic regression models to these feature sets and, for completeness, also fit lasso logistic regression and SVM models. We used default thresholds, and both norm-based and cross-validated hyperparameter settings. We summarize our results as follows:

- Feature selection often (although not always) improved the effectiveness of ridge logistic regression. BNS beat the other feature selection methods on macroaveraged $F 1$, so Table 3 shows results only for BNS. In no case, however, did feature selection increase the macroaveraged $F 1$ for ridge logistic regression on a data set to exceed that of lasso logistic regression with no feature selection.

- In no case did additional feature selection improve the macroaveraged $F 1$ of lasso logistic regression.

- Although it was not a focus of our experiments, we noted that feature selection sometimes improved the results of SVM models with default thresholds, but never improved the results of SVM models with tuned thresholds. SVMs built-in regularization is quite effective.
- Our use of cross-validation to choose the lasso hyperparameter on a per-category basis while using fixed feature set sizes for feature selection (the usual approach in text categorization research) perhaps gives an unfair unadvantage to lasso. Note, however, that lasso retains its dominance even with the (non-cross-validated) norm-based feature selection approach.

The number of features selected by the lasso varied from category to category but was always a small proportion of the full feature set. Figure 6 shows number of features chosen for each category on three collections; the other two collections are similar. The results shown in Figure 6 are with cross-validated hyperparameters. With the norm-based hyperparameter we found feature set sizes were 2-3 times larger: 2-892 (mean, 93.8; standard deviation [SD], 167.9) for ModApte, 0-3750 (mean, 625.9; SD, 726.9) for RCV1-v2, and 0-2636 (mean, 241.3; SD, 393.3) for OHSUMED. Thus cross-validation gives a substantial benefit in sparsity. Due to a limitation in the version of the software used for these experiments, the intercept term was penalized in the same fashion as other parameters. Unsurprisingly, in no case did lasso zero-out the parameter for the intercept term.

We found a strong correlation between the number of positive training examples and the number of features chosen. Figure 7 shows the relationship for the 90 categories and the 9,603 training examples of the ModApte collection. The other collections showed similar patterns. 
(a)

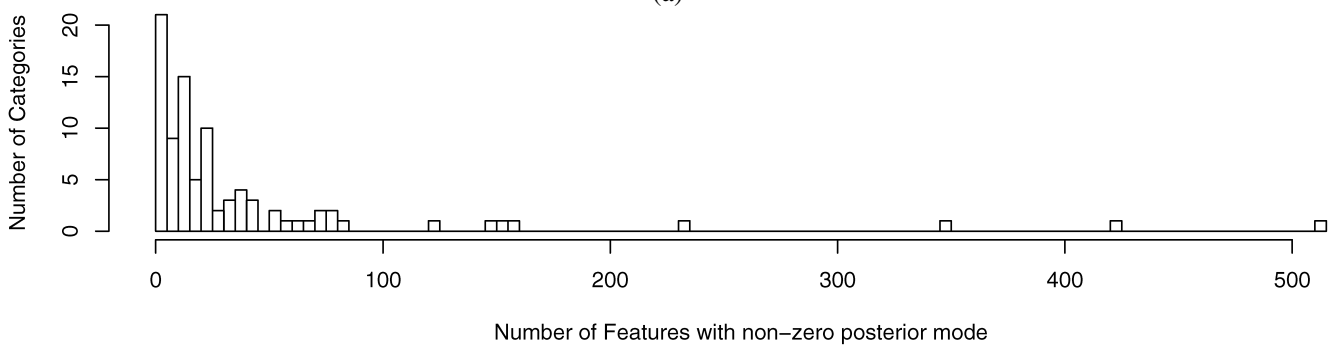

(b)

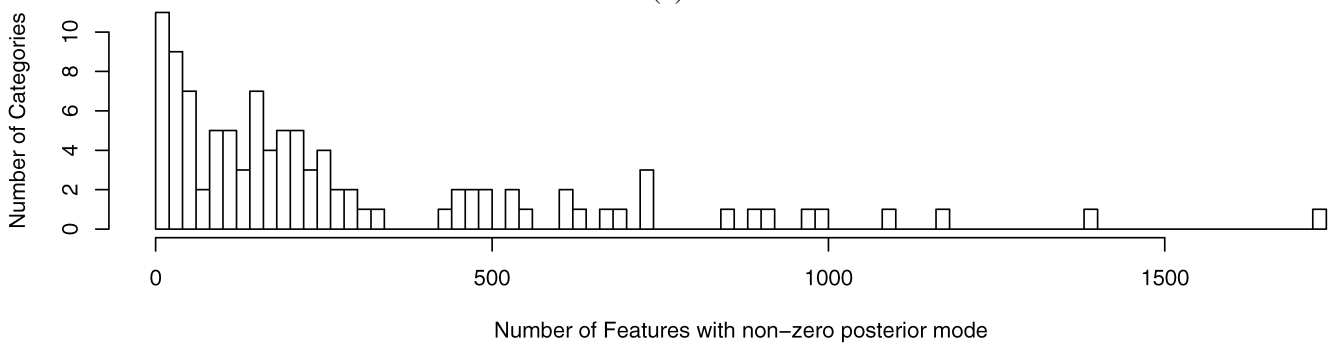

(c)

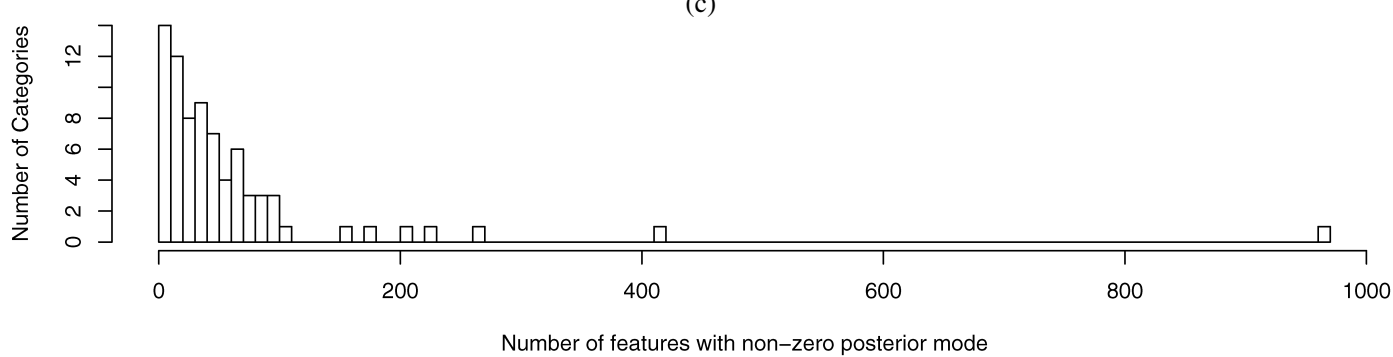

Figure 6. Number of features selected for each category in three test collections by lasso logistic regression (a) ModApte (21,989 features); (b) RCV1-v2 (47,152 features); (c) OHSUMED (122,076 features). Hyperparameter values were chosen by training set cross-validation.

In our examples, the SVM models included many more features than lasso models. For example, on the RCV1-v2 data set, lasso with cross-validated hyperparameters produced models with 3-1,737 features (mean, 294.1; SD, 328.6), whereas the SVM models had 299-28,481 features (mean, 8,414.5; SD, 5,859.6).

\section{SUMMARY}

Lasso logistic regression provides state-of-the-art text categorization effectiveness while producing sparse and thus efficient models. The approach is also useful in other highdimensional data analysis problems, such as predicting adverse drug events (Hauben, Madigan, Gerrits, and Meyboom 2005). Recent extensions broaden the range of applicability even further. We have already mentioned the extension to polytomous logistic regression, and other researchers have applied L1 regularization to more complex models (Li and Yang 2004).

Richer prior distributions also may prove useful. Those used in our experiments, although informative in the statistical sense, are not based on any knowledge of language or the meaning of categories. In recent work (Dayanik, Lewis, Madigan, Menkov, and Genkin 2006), we used textual descriptions of categories, user suggestions, and published reference materials to build priors that incorporate the knowledge that some features are likely to be more useful than others, providing significantly improved effectiveness.

Meinshausen (2005), Zhao and Yu (2006), and others have discussed the limitations of the lasso as a feature selection algorithm. Future work will explore alternatives such as Meinshausen's "relaxed lasso." Several algorithms that estimate "regularization paths" have emerged in recent years (see, e.g., Park and Hastie 2006). These algorithms have the attractive property of estimating coefficients for all possible hyperparameter values. However, scaling such algorithms to huge applications remains challenging.

\section{ACKNOWLEDGMENTS}

The U.S. National Science Foundation supported this work through grants EIA-0087022 (KD-D program), DMS-0113236 (ITR program), and DMS-0505599. The authors thank Andrei Anghelescu, Steve Benson, Aynur Dayanik, Susana Eyheramendy, Dmitriy Fradkin, Navendu Garg, Paul Kantor, Sathiya Keerthi, Paul Komarek, Vladimir Menkov, Fred Roberts, and Cun-Hui Zhang for suggestions, as well as Bill Hersh, Ken Lang, Andrew McCallum, the U.S. National Library of Medicine, Jason Rennie, and Reuters Ltd. for making available the data sets used in this research.

[Received January 2006. Revised September 2006.] 


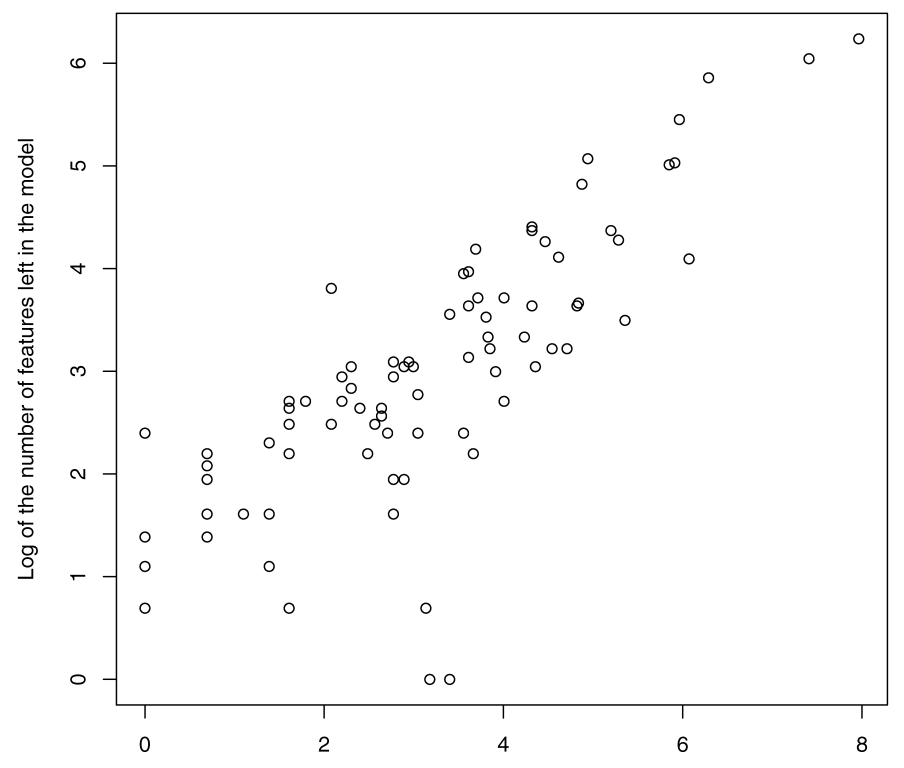

Log of the number of relevant documents in the training data

Figure 7. Number of relevant (positive) examples for a category versus number of nonzero parameters in fitted lasso model. Fitting was on all 9,603 ModApte training documents. Hyperparameters were chosen by cross-validation. Axes use logarithmic (base $e$ ) scales.

\section{REFERENCES}

Ackham, P. J. (2004), "An Algorithm for Computing the Inverse Normal Cumulative Distribution Function," available at http://home.online. no/ pjacklam/notes/invnorm/.

Berry, M. W. (ed.) (2004), Survey of Text Mining: Clustering, Classification, and Retrieval, New York: Springer-Verlag.

Dayanik, A., Lewis, D., Madigan, D., Menkov, V., and Genkin, A. (2006), "Constructing Informative Prior Distributions From Domain Knowledge in Text Classification," in Proceedings of SIGIR 2006: The Twenty-Ninth An nual International ACM SIGIR Conference on Research and Development in Information Retrieval, New York: ACM, pp. 493-500.

Dennis, J. E., Jr., and Schnabel, R. B. (1989), "A View of Unconstrained Optimization," in Optimization, eds. G. L. Nemhauser, A. H. G. Rinnooy Kan, and M. J. Todd, Amsterdam: Elsevier, pp. 1-72.

Efron, B., Hastie, T., Johnstone, I., and Tibshirani, R. (2004), "Least Angle Regression," The Annals of Statistics, 32, 407-499.

Figueiredo, M. A. T. (2003), "Adaptive Sparseness for Supervised Learning," IEEE Transactions on Pattern Analysis and Machine Intelligence, 25 1150-1159.

Figueiredo, M. A. T., and Jain, A. K. (2001), "Bayesian Learning of Sparse Classifiers," in Proceedings of the 2001 IEEE Computer Society Conference on Computer Vision and Pattern Recognition, 1, pp. I-35-I-41.

Forman, G. (2003), "An Extensive Empirical Study of Feature Selection Metrics for Text Classification," Journal of Machine Learning Research, 3, 1289-1305

Girosi, F. (1998), “An Equivalence Between Sparse Approximation and Support Vector Machines," Neural Computation, 10, 1455-1480.

Greenland, S., Schwartzbaum, J. A., and Finkle, W. D. (2000), "Problems From Small Samples and Sparse Data in Conditional Logistic Regression Analysis," American Journal of Epidemiology, 151, 531-539.

Hadjicostas, P. (2003), "Consistency of Logistic Regression Coefficient Estimates Calculated From a Training Sample," Statistics and Probability Letters, 62, 293-303.

Hastie, T. J., and Pregibon, D. (1992), "Generalized Linear Models," in Statistical Models in S, eds. J. M. Chambers and T. J. Hastie, Pacific Grove, CA: Wadsworth \& Brooks, pp. 377-420.

Hastie, T., Tibshirani, R., and Friedman, J. (2001), The Elements of Statistical Learning: Data Mining, Inference and Prediction, New York: Springer.

Hauben, M., Madigan, D., Gerrits, C., and Meyboom, R. (2005), "The Role of Data Mining in Pharmacovigilence," Expert Opinion in Drug Safety, 4, 929-948.

Hersh, W., Buckley, C., Leone, T. J., and Hickman, D. (1994), “OHSUMED: An Interactive Retrieval Evaluation and New Large Test Collection for Research," in SIGIR '94: Proceedings of the Seventeenth Annual International
ACM-SIGIR Conference on Research and Development in Information Retrieval, New York: ACM, pp. 192-201.

Hoerl, A. E., and Kennard, R. W. (1970), "Ridge Regression: Biased Estimation for Nonorthogonal Problems," Technometrics, 12, 55-67.

Jin, R., Yan, R., Zhang, J., and Hauptmann, A. (2003), "A Faster Iterative Scaling Algorithm for Conditional Exponential Models," in Proceedings of the Twentieth International Conference on Machine Learning (ICML-2003), Menlo Park: AAAI, pp. 282-289.

Joachims, T. (1998), "Text Categorization With Support Vector Machines: Learning With Many Relevant Features," in Machine Learning: ECML'98, 10th European Conference on Machine Learning, Heildelberg: Springer, pp. 137-142.

(2002), Learning to Classify Text Using Support Vector Machines, Boston: Kluwer.

Jurafsky, D., and Martin, J. H. (2000), Speech and Language Processing: An Introduction to Natural Language Processing, Computational Linguistics, and Speech Recognition, Englewood Cliffs, NJ: Prentice-Hall.

Kittler, J. (1986), "Feature Selection and Extraction," in Handbook of Pattern Recognition and Image Processing, eds. T. Y. Young and K.-S. Fu, Orlando, FL: Academic Press, pp. 59-83.

Kivinen, J., and Warmuth, M. K. (2001), "Relative Loss Bounds for Multidimensional Regression Problems," Machine Learning, 45, 301-329.

Komarek, P., and Moore, A. (2003), "Fast Robust Logistic Regression for Large Sparse Datasets With Binary Outputs," in Proceedings of the Ninth International Workshop on Artificial Intelligence and Statistics, pp. 197-204.

Krishnapuram, B., Hartemink, A. J., Carin, L., and Figueiredo, M. A. T. (2005), "Sparse Multinomial Logistic Regression: Fast Algorithms and Generalization Bounds," IEEE Transactions on Pattern Analysis and Machine Intelligence, 27, 957-968.

le Cessie, S., and van Houwelingen, J. C. (1997), "Ridge Estimators in Logistic Regression," Applied Statistics, 41, 191-201.

Li, F., and Yang, Y. (2004), "Recovering Genetic Regulatory Networks From Micro-Array Data and Location Analysis Data," Genome Informatics, 15, 131-140.

Lewis, D. D. (1995), "Evaluating and Optimizing Autonomous Text Classification Systems," in SIGIR'95: Proceedings of the 18th Annual International ACM SIGIR Conference on Research and Development in Information Retrieval, New York: ACM, pp. 246-254.

(1998), "Naive (Bayes) at Forty: The Independence Assumption in Information Retrieval," in Machine Learning: ECML'98, 10th European Conference on Machine Learning, Heildelberg: Springer, pp. 4-15.

(2004), "Reuters-21578 Text Categorization Test Collection: Distribution 1.0 README File (v 1.3)," available at http://www.daviddlewis.com/ resources/testcollections/reuters $21578 /$ readme.txt.

Lewis, D. D., Schapire, R. E., Callan, J. P., and Papka, R. (1996), “Training Algorithms for Linear Text Classifiers," in Proceedings of SIGIR-96, 19th ACM International Conference on Research and Development in Information Retrieval, New York: ACM, pp. 298-306.

Lewis, D. D., Yang, Y., Rose, T., and Li, F. (2004), "RCV1: A New Benchmark Collection for Text Categorization Research," Journal of Machine Learning Research, 5, 361-397. Data set available at http://jmlr.csail.mit.edu/ papers/volume5/lewis04a/lyrl2004_rcv1v2_README.htm.

Lowe, H. J., and Barnett, G. O. (1994), "Understanding and Using the Medical Subject Headings (MeSH) Vocabulary to Perform Literature Searches," Journal of the American Medical Association, 271, 1103-1108.

Luenberger, D. G. (1984), Linear and Nonlinear Programming (2nd ed.), Reading, MA: Addison-Wesley.

Madigan, D. (2005), "Statistics and the War on Spam," in Statistics: A Guide to the Unknown, eds. R. Peck, G. Casella, G. Cobb, R. Hoerl, D. Nolan, R. Starbuck, and H. Stern, Duxbury Press, pp. 135-147.

Madigan, D., and Ridgeway, G. (2004), Discussion of "Least Angle Regression," by B. Efron, T. Hastie, I. Johnstone, and R. Tibshirani, The Annals of Statistics, 32, 465-469.

Madigan, D., Genkin, A., Lewis, D. D., Argamon, S., Fradkin, D., and Ye, L. (2005a), "Author Identification on the Large Scale," in Proceedings of the CSNA \& INTERFACE Annual Meetings, CD-ROM.

Madigan, D., Genkin, A., Lewis, D. D., and Fradkin, D. (2005b), "Bayesian Multinomial Logistic Regression for Author Identification," in Bayesian Analysis and Maximum Entropy Methods in Science and Engineering: 25th International Workshop on Bayesian Inference and Maximum Entropy Methods in Science and Engineering, AIP Conference Proceedings, Vol. 803, Melville, NY: AIP, pp. 509-516.

Mallick, B. K., Ghosh, D., and Ghosh, M. (2005), "Bayesian Classification of Tumors Using Gene Expression Data," Journal of the Royal Statistical Society, Ser. B, 67, 219-234.

Malouf, R. (2002), "A Comparison of Algorithms for Maximum Entropy Parameter Estimation," in Proceedings of the Sixth Conference on Natural Language Learning (CoNLL-2002), pp. 49-55. 
Manning, C., and Schütze, H. (1999), Foundations of Statistical Natural Language Processing, Cambridge, MA: MIT Press.

Maron, M. E. (1961), "Automatic Indexing: An Experimental Inquiry," Journal of the ACM, 8, 404-417.

Meinshausen, N. (2005), "Lasso With Relaxation," technical report, University of California Berkeley, Dept. of Statistics.

Mitchell, T. J., and Beauchamp, J. J. (1988), "Bayesian Variable Selection in Linear Regression," Journal of the American Statistical Association, 83, $1023-1032$.

Mosteller, F., and Wallace, D. L. (1964), Inference and Disputed Authorship: The Federalist, Addison-Wesley.

Park, M.-Y., and Hastie, T. (2006), "An L1 Regularization-Path Algorithm for Generalized Linear Models," available at http://www-stat.stanford.edu/ hastie/Papers/glmpath.pdf.

Pike, M. C., Hill, A. P., and Smith, P. G. (1980), "Bias and Efficiency in Logistic Analysis of Stratified Case-Control Studies," American Journal of Epidemiology, 9, 89-95.

Porter, M. F. (1980), "An Algorithm for Suffix Stripping," Program, 14 (3), $130-137$.

(2003), "The Porter Stemming Algorithm," available at http://www. tartarus.org/martin/PorterStemmer/index.html.

Press, W. H., Teukolsky, S. A., Vetterling, W. T., and Flannery, B. P. (1992), Numerical Recipes in C: The Art of Scientific Computing (2nd ed.), Cambridge, U.K.: Cambridge University Press.

Rocchio, J. J., Jr. (1971), "Relevance Feedback Information Retrieval," in The SMART Retrieval System: Experiments in Automatic Document Processing, ed. G. Salton, Englewood Cliffs, NJ: Prentice-Hall, pp. 313-323.

Salton, G., and Buckley, C. (1988), "Term-Weighting Approaches in Automatic Text Retrieval," Information Processing and Management, 24, 513-523.

Santner, T., and Duffy, D. (1989), The Statistical Analysis of Discrete Data, New York: Springer-Verlag.

Schapire, R. E., and Singer, Y. (2000), "BoosTexter: A Boosting-Based System for Text Categorization," Machine Learning, 39, 135-168.
Sebastiani, F. (2002), "Machine Learning in Automated Text Categorization," ACM Computing Surveys, 34, 1-47.

Shevade, S. K., and Keerthi, S. S. (2003), "A Simple and Efficient Algorithm for Gene Selection Using Sparse Logistic Regression," Bioinformatics, 19, 2246-2253.

Smith, R. L. (1999), "Bayesian and Frequentist Approaches to Parametric Predictive Inference" (with discussion), in Bayesian Statistics 6, eds. J. M. Bernardo, J. O. Berger, A. P. Dawid, and A. F. M. Smith, Oxford, U.K.: Oxford University Press, pp. 589-612.

Tibshirani, R. (1996), "Regression Shrinkage and Selection via the Lasso," Journal of the Royal Statistical Society, Ser. B, 58, 267-288.

Tipping, M. E. (2001), "Sparse Bayesian Learning and the Relevance Vector Machine," Journal of Machine Learning Research, 1, 211-244. Rijsbergen

van Rijsbergen, C. J. (1972), "Automatic Information Structuring and Retrieval," unpublished doctoral thesis, King's College, Cambridge. (1979), Information Retrieval (2nd ed.), London: Butterworths.

Weiss, S. M., Indurkhya, N., Zhang, T., and Damerau, F. J. (2005), Text Mining: Predictive Methods for Analyzing Unstructured Information, New York: Springer.

Yang, Y., and Pedersen, J. O. (1997), "A Comparative Study on Feature Selection in Text Categorization," in Proceedings of ICML-97, 14th International Conference on Machine Learning ICML97, San Francisco: Morgan Kaufmann, pp. 412-420.

Zhang, J., and Yang, Y. (2003), "Robustness of Regularized Linear Classification Methods in Text Categorization," in Proceedings of SIGIR 2003: The Twenty-Sixth Annual International ACM SIGIR Conference on Research and Development in Information Retrieval, New York: ACM, pp. 190-197.

Zhang, T., and Oles, F. (2001), "Text Categorization Based on Regularized Linear Classifiers," Information Retrieval, 4, 5-31.

Zhao, P., and Yu, B. (2006), "On Model Selection Consistency of Lasso," Journal of Machine Learning Research, 7, 2541-2567.

Zou, H., and Hastie, T. (2005), "Regularization and Variable Selection via the Elastic Net," Journal of the Royal Statistical Society, Ser. B, 67, 301-320. 